\title{
Modelling adapted to manufacturing aspects of holographic grating structures
}

\section{O. Sandfuchs \\ sandfuchs@zeiss.de \\ C. Schwanke}

\section{Burkhardt}

\section{F. Wyrowski}

\section{A. Gatto}

\section{R. Brunner}

\author{
Technology Microstructured Optics, Carl Zeiss Jena GmbH, Carl-Zeiss-Promenade 10, 07740 Jena, \\ Germany \\ Institute of Applied Physics, Friedrich-Schiller-Universität, Albert-Einstein-Str. 15, 07745 Jena, \\ Germany \\ Fraunhofer Institute for Surface Engineering and Thin Films, Bienroder Weg 54 E, 38108 Braunschweig, \\ Germany \\ Technology Microstructured Optics, Carl Zeiss Jena GmbH, Carl-Zeiss-Promenade 10, 07740 Jena, \\ Germany \\ Institute of Applied Physics, Friedrich-Schiller-Universität, Albert-Einstein-Str. 15, 07745 Jena, \\ Germany \\ Technology Microstructured Optics, Carl Zeiss Jena GmbH, Carl-Zeiss-Promenade 10, 07740 Jena, \\ Germany \\ Technology Microstructured Optics, Carl Zeiss Jena GmbH, Carl-Zeiss-Promenade 10, 07740 Jena, \\ Germany \\ University of Applied Sciences Jena, Carl Zeiss Promenade 2, 07745 Jena, Germany
}

The diffraction efficiencies of modified sinusoidal and blazed gratings are investigated in the high spatial frequency regime by rigorous numerical methods and are compared to experimentally manufactured gratings. The introduced modifications take actual technological induced variations of the profile geometries, such as specific corner rounding, into account. The high spatial frequency regime (resonance regime) is characterized by a local grating period, $g$, to wavelength, $\lambda$, ratio of $0.7 \leq \mathrm{g} / \lambda \leq 4$ and shows an important relevance for applications in spectroscopy and diffractive imaging. The investigations are carried out for both reflection on metallic surfaces and transmission of dielectric structures over a broad range of grating periods and incidence angles. It was found that near the grating resonance, the more simply producible sine gratings can compete in diffraction efficiency with sawtooth structures. Additionally, for certain application conditions, holographically modified sine structures achieve higher efficiencies than the ideal sine profile. It is also shown that holographic sinusoidal-like profiles measured by AFM can be fitted to a super-Caussian shape, which is then used to inversely reconstruct the structure profiles from efficiency data. [D0I: 10.2971/jeos.2011.11006]

Keywords: diffraction gratings, holographic manufacturing, resist simulation, efficiency-performance optimization, structural and optical characterization

\section{INTRODUCTION}

Diffraction gratings are key components for both spectrally analyzing and imaging optical systems. For applications such as miniaturized spectrometers, hybrid objectives for fluorescence microscopy or semiconductor inspection systems, the optical design aims at achieving a high efficiency of the diffractive component [1]-[6]. Commonly, theoretical predictions rely on ideal grating structures, such as a perfect triangular sawtooth (blazed grating) or a sinusoidal profile. However, manufacturing processes such as interference lithography often result in deviations from the perfect shape and induce features such as corner rounding in the surface profile. To derive quantitative predictions of the interactions between the incoming light and the grating structure, different appropriate approaches can be distinguished which mainly depend on the ratio of the grating period $g$ to the wavelength $\lambda$. The scalar theory allows an appropriate treatment for the regime $g / \lambda>100$ in which the blazed gratings possess the highest diffraction efficiency. The intermediate range $4 \leq g / \lambda \leq 100$ is characterized by the transition from the strictly rigorous regime to the validity of the scalar theory (see Figure 1). Here, electromagnetic shadowing effects have to be considered and, for blazed gratings, lead to a diffraction efficiency reduction which obeys a linear dependence on $g / \lambda[7,8]$.

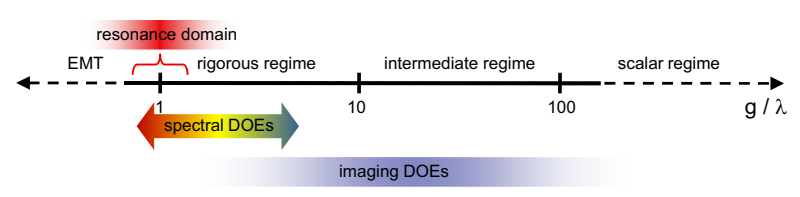

FIC. 1 Different diffraction regimes depending on the grating structure size, i.e. the grating period g scaled to the wavelength $\lambda$. General fields of application of the DOES are indicated. 
In this contribution we focus on the high spatial frequency range $0.7 \leq g / \lambda \leq 4.0$ around the resonance domain. This regime is of importance for many advanced spectroscopic gratings and also for selected diffractive imaging elements. Hereby the problem of gaining a high diffraction efficiency in the first diffraction order is more advanced and repeatedly raises the question of how to choose a proper grating structure. Especially ideal geometric structures, such as perfect triangular blaze profile or ideal sine, show similar efficiencies in different settings, so that a clear optical advantage of one geometrical structure is not immediately evident. This fact has an important impact on practical issues because certain surface geometries (e.g. sine structure) are more advantageous with regard to feasibility and reproducibility during mastering and replication than others (e.g. perfect sawtooth grating). Additionally, in this high frequency regime, actual technological induced deviations from the ideal structure become relevant, e.g. leading to corner rounding in blazed profiles or to asymmetric fill factors of the sinusoidal-like structures.

We perform rigorous optimization of ideal sawtooth and sine gratings in the resonance regime for various design parameters, including the angle of incidence $\theta$ and the scaled grating period $g / \lambda$, where we have used an integral equation method [9]. Hereby both aspects are considered, the reflection on metallic surfaces and the transmission of dielectric structures. For the optimization in the multiple parameter space we implemented a downhill-simplex-algorithm in our analysis [10].

The last section of the paper focuses on optical effects caused by topographic deviations from the ideal sine profile, which are induced by the manufacturing process of the gratings. To describe the influence of the manufacturing process on the realized surface topography, interference lithography (IL) was applied as a model technology. In the IL process, a resist coated substrate is exposed to an interference fringe system generated by coherent wave fronts. During the recording process, a latent structure is created in the resist, which is transformed into a continuous surface phase profile in the subsequent development process [11]. Although the amplitude distribution of the interference field shows a sine structure, the following necessary process steps determine a nonlinear transfer, which results in a modified sinusoidal-like structure. Based on an analytical model of the IL [12] we show that a beneficial use of the process parameters allows the realization of modified surface profiles with an increased efficiency performance compared to perfect sine structures.

The theoretical results for the grating structures are compared to real gratings, which have been manufactured using the IL process. We structurally and optically characterize the gratings and show that the sinusoidal-like profiles both obtained from Mello's resist model [13] and by AFM measurements can be fitted by super-Gaussian shapes. Moreover, one disadvantage of the optimization using ideal profiles, which we have overcome, is that it does not consider the inherent dependency of the fill factor and the aspect ratio, which we have found for holographic profiles. Finally, we address another question that repeatedly arises during characterization of optical gratings: "What structure is actually seen by the light?" Based on the super-Gaussian fit, we perform an inverse reconstruction of profiles from efficiency data.

\section{BLAZING OF IDEAL GRATINGS NEAR RESONANCE}

High-frequency grating structures in the resonance domain with periods close to the wavelength of the light, $0.7<g / \lambda<4.0$, possess only a few diffraction orders, in which the outgoing light is coupled. The notion "resonance domain" refers both to the structural resonance with the light wavelength but also to the fact that in this region an extraordinary high diffraction efficiency can be achieved as a consequence of a Bragg-resonance phenomenon. To achieve this maximum efficiency for a single order, the other orders have to be suppressed by an appropriate choice of profile geometry in accordance with the angle of incidence $\theta$.

Regarding the manufacturing of master DOEs and their replication, simple profile structures are preferred. Most spectrometer modules commonly involve blazed or sine gratings. However, due to the specific manufacturing process, such as IL, real grating profiles often deviate from these ideal structures. First, we start by considering two ideal profile shapes: perfect triangular (Figure 2(a)) and perfect sinusoidal profiles (Figure 2(b)). We optimize their corresponding structure parameters by numerical simulations. Second, we exploit a twowave theory [14] to explain the influence of real manufactured profile shapes on the efficiency performance in the resonance domain.

The triangular grating possesses two independent geometry

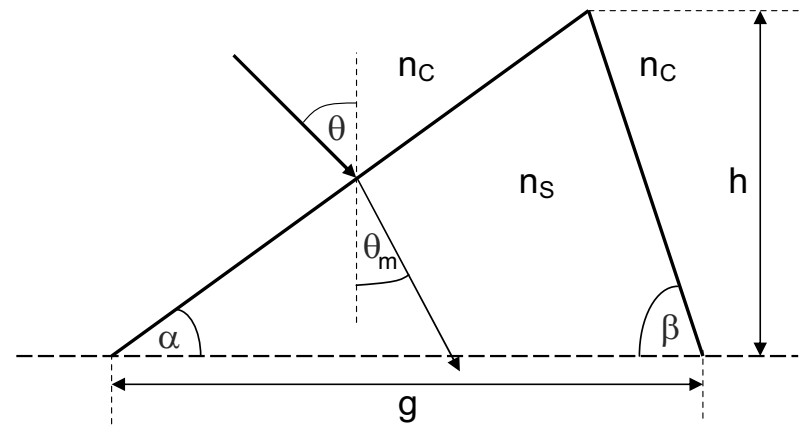

FIG. 2a Profile geometries of two grating structures: triangular blaze.

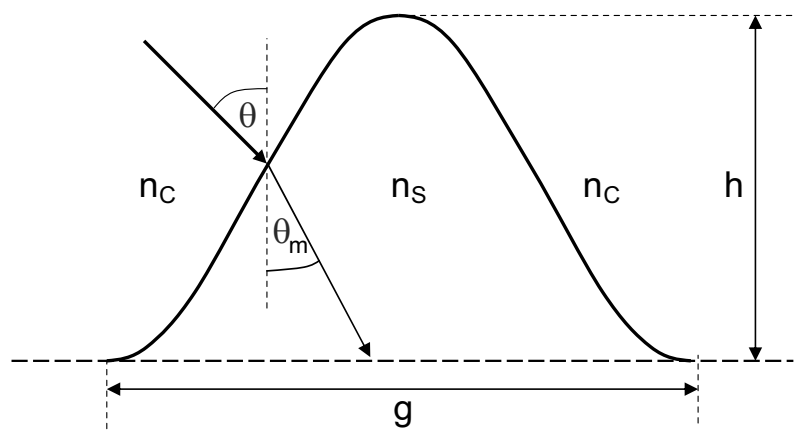

FIG. 2b Profile geometries of two grating structures: ideal sine. 


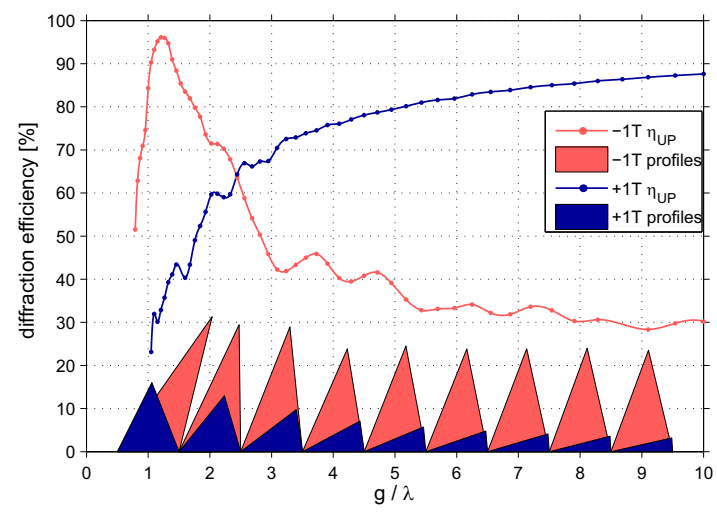

FIG. 3 Optimized efficiency of triangular profiles in transmission geometry, for normal incidence $(\theta=0)$ and random polarization, i.e. unpolarized (UP) light. The corresponding profile shapes are indicated at each integer value of $g / \lambda$ depicting the aspect ratio of the structure. The wavelength is $\lambda=550 \mathrm{~nm}$ and refractive index $n=1.5$.

parameters: the angle $\alpha$ for active blaze facet and $\beta$ for the passive facet. Whereas the ideal sine profile has only one degree of freedom: its profile height $h$.

From rigorous diffraction calculations one finds the wellknown blazing (blue curve in Figure 3 ) in the $1^{\text {st }}$ diffraction order for the triangular dielectric transmission gratings. Reducing the structure period the blaze efficiency decreases because of electromagnetic shadowing effects, e.g. [15]. In the same work it has been shown that by adjusting the passive blaze facet shadowing can be partially avoided. However, for $g / \lambda<2.5$ and $\lambda=550 \mathrm{~nm}$ and $n=1.5$ the efficiency inevitably drops below $65 \%$. At the same grating period, the efficiency in the -1st diffraction order has increased above $65 \%$ and reaches its maximum of $\sim 95 \%$ at about $g / \lambda=1.15$ (red curve in Figure 3). It is caused by a Bragg-resonance effect, which has been explained by Golub et al. [14]. Also note that in Figure 3 the data points have been calculated while optimizing the structure profile for each grating period separately. The corresponding profile shapes are displayed above the abscissa. Thus the maximum energy diffracted by the structure eventually flips from the $+1^{\text {st }}$ to the $-1^{\text {st }}$ diffraction order.

A systematic optimization for the triangular profile within the parameter space containing the angle of incidence $\theta$ and scaled grating period $g / \lambda$, reveals that there is an optimum grating period with high efficiency around $g / \lambda=1.1 \ldots 1.2$ for all angles of incidence considered $\left(\theta=0^{\circ} \ldots 40^{\circ}\right)$. Again, at each data point in Figure 4, the profile has been optimized and the active and passive angles have been adjusted. It becomes clear that, depending on the symmetry given by $\theta$, the profile itself has to be adapted to this symmetry. This procedure is of course different for the sine profile (cf. Figure 2(b)), because the sine is a symmetric profile for any height. Consequently, the asymmetry can only be introduced by a slanted angle of incidence. In the rigorous simulations, the optimum angle is attained close to the Bragg-incidence condition (white dashed line in Figure 4(b)) and attains its maximum value at $95 \%$ for $g / \lambda=1.23$ and $\theta=23.9^{\circ}$ and an optimum height of $h=1.83^{*} \mathrm{~g}=1.23 \mu \mathrm{m}$. The efficiency of the sine profile is as large as the maximum efficiency for the corresponding triangular profile in Figure 4(a).

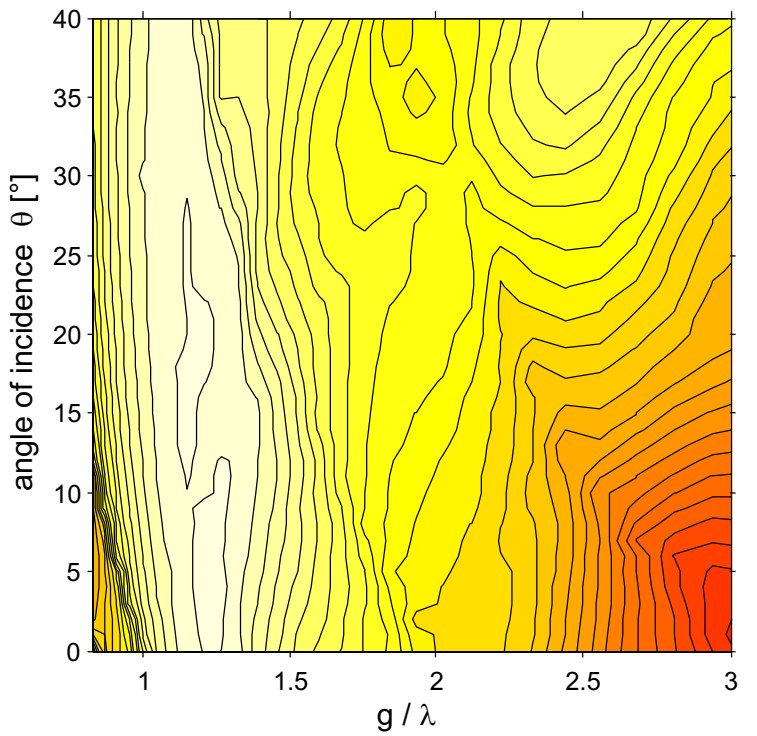

FIG. 4a Efficiency of unpolarized light in the -1st diffraction order obtained by a rigorous optimization of the two types of gratings: triangular profile with refractive index $\mathrm{n}=1.5$.

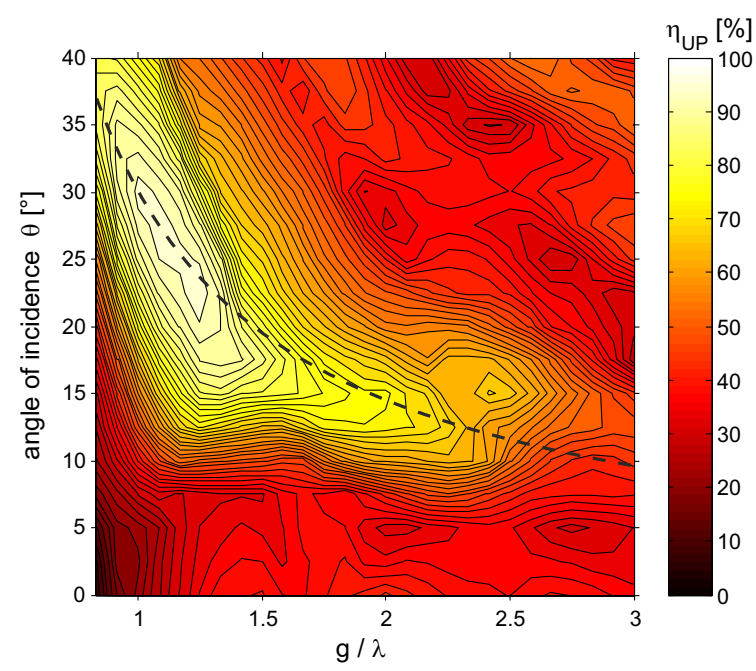

FIC. 4b Efficiency of unpolarized light in the -1st diffraction order obtained by a rigorous optimization of the two types of gratings: sinusoidal profile with refractive index $\mathrm{n}=1.5$.

This behaviour of maximum efficiency in the resonance domain seems to occur independently of the actual profile structure, here shown by rigorous calculations for the triangle or the sine. To understand this result, we exploit the two-wave theory by Golub et al. [14], in which he explained an unusually high diffraction efficiency in the resonance domain by applying the Kogelnik theory of Bragg structures [16, 17] to surface relief gratings in transmission geometry and was able to derive an analytical efficiency formula.

In the following, we will briefly summarize Golub's two-wave theory as far as it is necessary to discuss the numerical and experimental results of our paper. The two-wave theory is valid for slanted and unimodal profiles, i.e. profiles having only a single maximum. The profile is expressed as a sinusoidal graded-index modulation embedded in an averaged in- 
(a)

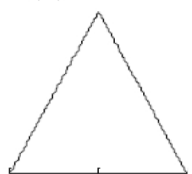

(b)

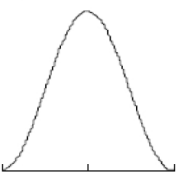

(c)

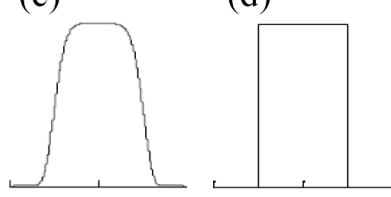

FIG. 5 Typical grating structures all possessing the same areal duty cycle of $f=0.5$ : (a) triangular, (b) sinusoidal, (c) super-Gaussian, and (d) rectangular.

dex background, and is then expanded in a Fourier series:

$$
n\left(\chi_{r}\right)^{2}=\bar{n}^{2}+\left(n_{S}^{2}-n_{C}^{2}\right) \times \sum_{m=-\infty}^{+\infty} G_{m} e^{2 \pi i m \chi_{r}}
$$

Where $\chi_{r}$ is the abscissa scaled to the grating period and $G_{m}$ are the Fourier coefficients of the symmetric profile structure, after the profile has been symmetrized by a rotation with the inverse slant angle $-\varphi$. The slant angle $\varphi$ is, in general, defined independently of the specific profile structure as

$$
\tan \varphi=\left(\chi_{a}-0.5\right) \frac{g}{h}
$$

where the scaled coordinate $\chi_{a}$ marks the foot of the profile's crest, and thus $\tan \varphi$ is always inversely proportional to the profile height $h$. The averaged refractive index is

$$
\bar{n}^{2}=n_{C}^{2}+\left(n_{S}^{2}-n_{C}^{2}\right) f
$$

where $f$ is the areal duty cycle of the profile structure, and $n_{C}$ and $n_{S}$ are the refractive indices of the superstrate (cladding) and the substrate, resp. Using a generalized Bragg condition for slanted surface relief structures, the angle of incidence $\theta_{B}$ for achieving an optimal efficiency is given by

$$
\sin \theta_{B}=\frac{(-m) \lambda}{2 n_{C} g}-\tan \varphi \sqrt{\frac{\left(\bar{n} / n_{C}\right)^{2}}{1+\tan ^{2} \varphi}-\left(\frac{m \lambda}{2 n_{C} g}\right)^{2}} .
$$

The diffraction efficiency for the $-1^{\text {st }}$ order then yields

$$
\eta_{T E, T M}(h, \lambda, \theta)=\frac{\sin ^{2}\left(\Gamma_{T E, T M} \times \sqrt{1+\Omega^{2}}\right)}{1+\Omega^{2}} .
$$

Where the parameters $\Gamma$ and $\Omega$ depend on the TE- and TMpolarization and are defined as

$$
\begin{aligned}
\Omega(\lambda, \theta) & =\frac{2 m \Lambda}{\hat{n} G_{1}}(\sin \theta+m \Lambda) \\
\Gamma_{T E, T M}(h, \lambda, \theta) & =\frac{\pi h G_{1}}{\lambda} \frac{\hat{n} \kappa_{T E, T M}}{\sqrt{1-\sin ^{2} \theta+\hat{n} f}} \\
\kappa_{T E} & =1 \\
\kappa_{T M} & =1-\frac{2 m^{2} \Lambda^{2}}{1+\hat{n} f} .
\end{aligned}
$$

With the scaled wavelength $\Lambda=\lambda /\left(2 n_{C} g\right)$ and scaled refractive index $\hat{n}=\left(n_{S}^{2}-n_{C}^{2}\right) / n_{C}^{2}$. For simplicity within this work, the parameters Eqs. (6)-(8) have been given in the case of a symmetric profile $(\varphi=0)$, so that the efficiency follows a sinefunction with respect to the profile height $h$ and follows a sinc-function with respect to the wavelength $\lambda$ and incidenceangle $\theta$, resp. The efficiency given by Eq. (5) is, in general, valid for any slant angle. From the two-wave theory it follows immediately that different profile structures as depicted in Figure 5 possess the same diffraction efficiency if they have the same areal duty cycle $f$. However, the corresponding optimal structure height $h_{\text {opt }}$ to achieve the maximum efficiency is different and obeys the following simple relation:

$$
h_{\text {opt }} \times\left|G_{1}\right|=\text { const. }
$$

Here $G_{1}$ is the first Fourier coefficient of the symmetrized profile. To put it in other words: in the resonance domain, the light "sees" only the first Fourier component of the dielectric grating structure:

\begin{tabular}{|c|c|}
\hline profile structure & $\left|\mathbf{G}_{1}\right|$ \\
\hline trinagular & $2 / \pi^{2}=0.203$ \\
\hline sinusoidal & $1 / 4=0.250$ \\
\hline super-Gaussian $(\mathrm{n}=6)$ & 0.301 \\
\hline rectangular & $1 / \pi=0.318$ \\
\hline
\end{tabular}

TABLE 1 Fourier coefficients $G_{1}$ of four typical profile structures as in Figure 5.

As an immediate consequence, in the resonance domain deviations from the ideal profile shape have only little influence on the maximum efficiency. They may slightly increase the light scattered into unwanted diffraction orders. The rectangular profile, e.g., has the largest Fourier coefficient and, consequently, the smallest optimum height. The rigorous efficiency is, however, a few percent smaller than predicted by the theory, because the rectangle has a predominant flat top, so light will be reflected more than for the triangle. This effect is not captured by the two-wave theory [14]. A maximum efficiency of $100 \%$ (neglecting Fresnel losses) is possible at optimum height $h_{\text {opt }}$, only for each polarization state, separately. The generalized Bragg condition (Eq. (4)) relates the asymmetry given by the slant angle of the profile to the remaining asymmetry provided by the optimum angle of incidence necessary for a maximum efficiency.

The assumption of the two-wave theory is strictly speaking only fulfilled for $g / \lambda \leq 1.5$. However, even away from the Bragg-incidence condition, we find that the two-wave theory gives a rather good approximation of the efficiency performance both for $\lambda$ and for $\theta$, as long as the higher diffraction orders do not gain too much energy (Figure 6).

Note that the slant angle $\varphi=\varphi(h)$ is in general dependent on the height (Eq. (2)), so that the solution for the optimum height $h_{\text {opt }}$ from the requirement $\eta=100 \%$ (cf. Eq. (5)) leads to a biquadratic equation for $h$ in the case of TE-polarization and to a bi-cubic equation in the case of TM-polarization, whose solutions have to be determined by finding the corresponding zeros. Only for symmetric profiles $(\varphi=0), h_{o p t}$ can be expressed completely in an analytical form.

Most spectrometer applications use gratings in reflection geometry. Similar to the optimization as in Figure 4 for transmission geometry, we now perform a rigorous efficiency optimization for metallic triangle and sine structures in reflection geometry (Figure 7). 


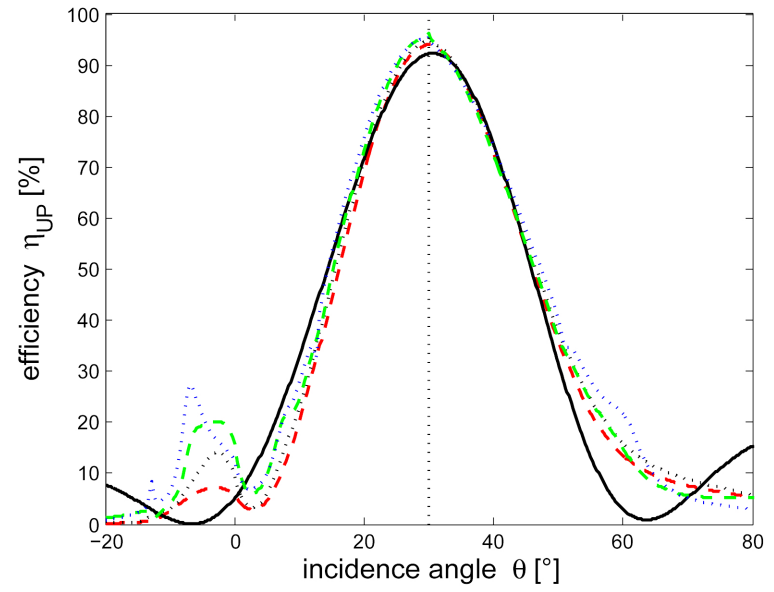

FIC. 6a Comparison of rigorous calculations for 4 different symmetric profile types: triangle, sine, super-Gaussian $(n=3)$ and rectangle with the two-wave theory (2WT) for sine profile near the Bragg-resonance condition $(g / \lambda=1.0)$ at each corresponding optimum height $h_{\text {opt }}$, depending on incidence angle.

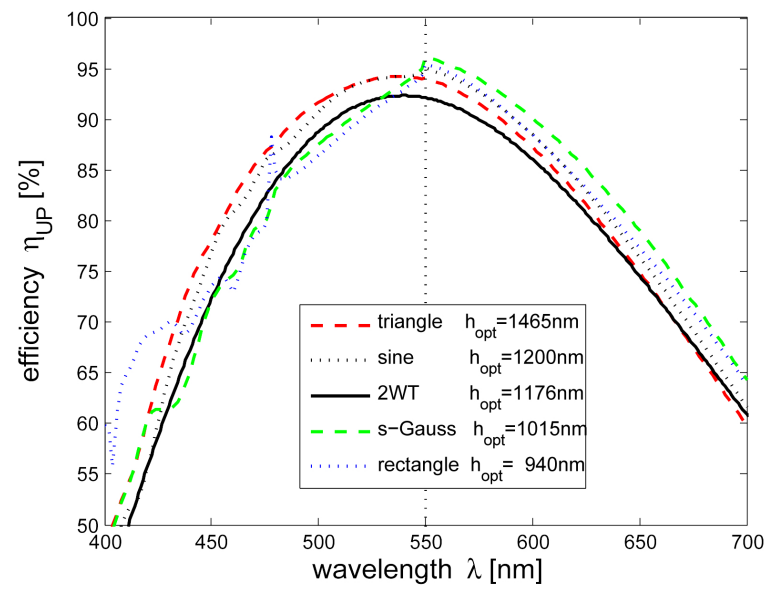

FIG. 6b Comparison of rigorous calculations for 4 different symmetric profile types: triangle, sine, super-Gaussian $(n=6)$ and rectangle with the two-wave theory (2WT) for sine profile near the Bragg-resonance condition $(g / \lambda=1.0)$ at each corresponding optimum height $h_{\text {opt }}$, depending on wavelength.

The efficiency behaviour of both profile types shows maximum values along the Littrow-incidence condition $\sin \theta_{L}=-m \lambda /\left(2 n_{C} g\right)$ (dashed black lines in Figure 7$)$. In this parameter space only the $-1^{\text {st }}$ and $0^{\text {th }}$ orders propagate. The resonance domain in the reflection geometry shows pronounced boundaries, which arise from the occurrence of the $+1^{\text {st }}$ and $-2^{\text {nd }}$ diffraction orders. The triangular profile has a global maximum at $g / \lambda=1.3$ and $\theta=22.5^{\circ}$ achieving a maximum efficiency of $83 \%$ for $\alpha=25^{\circ}$ and $\beta=86^{\circ}$ (corresponding to a structure height of $h=312.5 \mathrm{~nm}$ ), and the sine achieves $75 \%$ at an even lower height $h=230 \mathrm{~nm}$. The global maximum efficiency for the sine is attained at $g / \lambda=1.4$ and $\theta=20.9^{\circ}$ with an efficiency of $80 \%$ for $h=300 \mathrm{~nm}$.

From our rigorous calculations it has become clear that under the Littrow mount both triangular and sinusoidal grating structures show almost equally high efficiencies, if the incidence angle is chosen to break the symmetry accordingly. The structure height of the optimum sine can be smaller than for the triangular profile, which has advantages for the manufac-

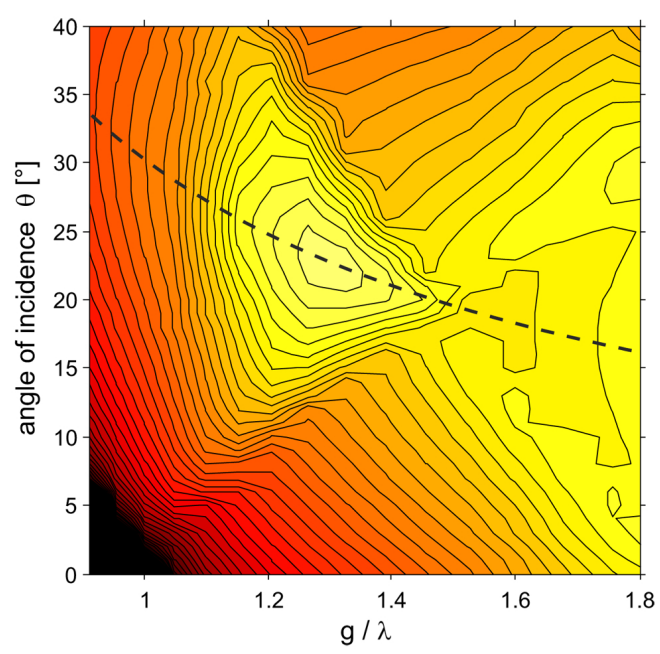

FIG. 7a Efficiency of unpolarized light in the -1st diffraction order obtained by a rigorous optimization of the two types of aluminum gratings: triangular profile in reflection with refractive index $\mathrm{n}_{S}(\lambda=550 \mathrm{~nm})=0.96+6.69$ i. Dashed black lines: Littrow-incidence condition.

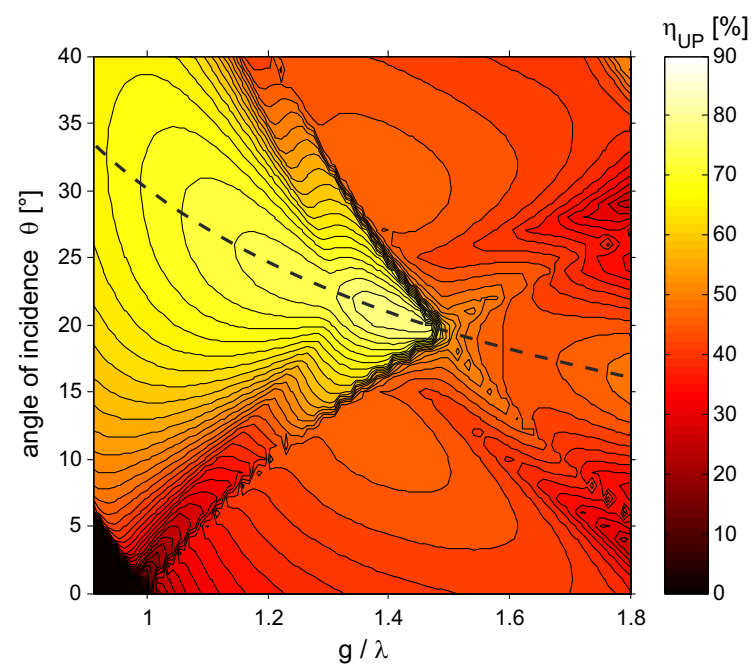

FIG. 7b Efficiency of unpolarized light in the -1st diffraction order obtained by a rigorous optimization of the two types of aluminum gratings: sinusoidal profile in reflection with refractive index $n_{S}(\lambda=550 \mathrm{~nm})=0.96+6.69$ i. Dashed black lines: Littrowincidence condition.

turing process. So, even for metallic structures, similarities are present to the results of the two-wave theory, although it is strictly speaking only applicable in the case of dielectric gratings. For the reflection geometry, up to now there exists, however, no analytical wave theory capable of describing this resonance phenomenon in an analogous way to the theory by Golub for the transmission geometry.

\section{INTERFERENCE LITHOGRAPHY AND REAL GRATING STRUCTURES}

Interference lithography (IL) is a well-established method for the microstructuring of optical surfaces. Especially, IL is a standard technique to fabricate spectroscopic gratings [1, 12] and it is also suitable for making imaging diffractive optical elements [4]-[6] or beam shaping components [11]. In the IL 


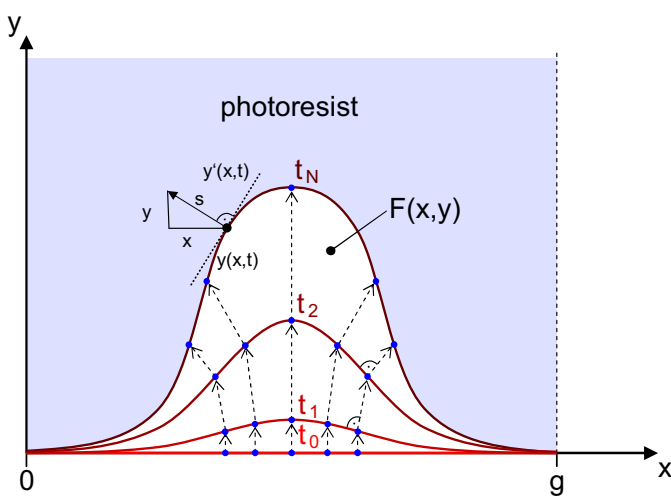

FIG. 8 Temporal evolution of a sinusoidal illumination in the photoresist.

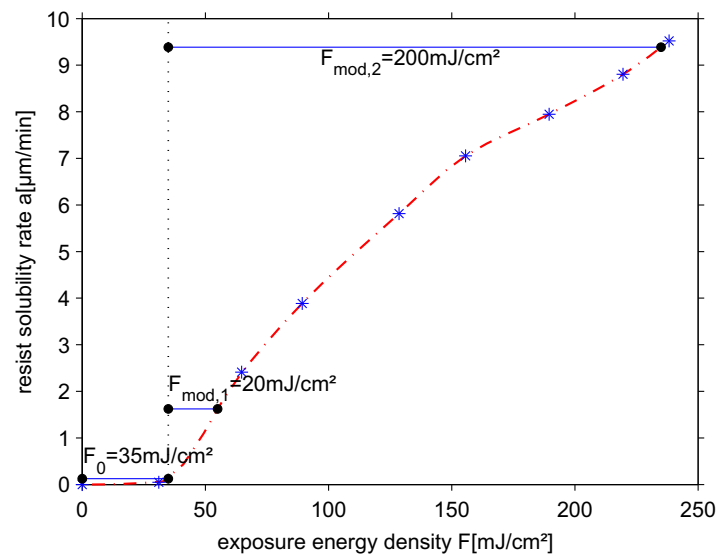

FIG. 9 Experimentally measured response curve of the photoresist AZ1518. Two different energy densities $\mathrm{F}_{\text {mod }}$ are marked.

process, a resist coated substrate is exposed to a stationary interference fringe system generated by coherent wave fronts. During the recording process, a latent structure is created in the resist, which is transformed into a continuous surfacerelief profile in the subsequent development process. The period $\gamma$ of the intensity variation is given by $\gamma=\lambda_{\text {rec }} /(2 \sin \psi)$, where $\lambda_{\text {rec }}$ is the recording wavelength and $\psi$ is half the angular separation of the intersecting waves. It is related to the grating period $g=\gamma / \cos \xi$ by the projection angle $\xi$ of the interference pattern onto the substrate [11]. The resulting relief profile recorded in the photoresist depends on both the exposure light pattern through the film and on the complete response of the photoresist, including the development process. The orientation of the exposure light pattern with respect to the photoresist is characterized by the bisector of the intersecting wave fronts. In general, the photoresist is aligned obliquely to the fringe pattern so that the alternating soluble and insoluble layers are inclined within the bulk of the resist.

Different simulation models have been introduced to allow a prediction of the emerging surface relief structure during the wet development process. For our investigations we apply a model introduced by Mello et al. [13]. Here, the photoresist response curve is an essential input parameter, describing the dissolution rate as a function of the exposure dose. For simplicity and with respect to our experimental setup, we neglect bleaching of the resist and multiple light reflections within the

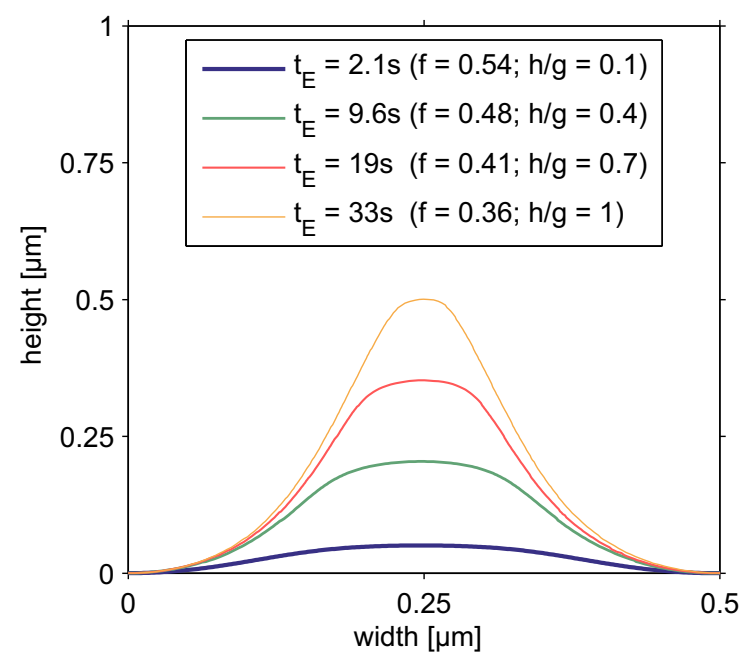

FIG. 10a Profile shapes obtained for four development times and two different energy doses: $20 \mathrm{~mJ} / \mathrm{cm}^{2}$ with increasing development time $\mathrm{t}_{E}$ (from lower to upper curves).

resist-substrate-layer system. Following Mello et al. the surface structure $y(x, t)$ can be calculated from the exposure light distribution $F(x, y)$ and the resist response curve $a(F)$ :

$$
\frac{\partial y}{\partial t}(x, t)=\frac{a(F[x, y(x, t)])}{\sqrt{1+\left(\frac{\partial y}{\partial x}(x, t)\right)^{-2}}} .
$$

Here, $y$ is the height of the structure at a lateral position $x$ and at a time $t$ after starting the development process. As an example, Figure 8 shows the schematic time evolution of the surface profile of an exposed sinusoidal grating structure. Even with the assumption of a linear resist response curve, the isotropy of the wet development produces a narrowing of the top of the structures so that the resulting profile differs from the original sinusoidal pattern in the photoresist. This is due to the fact that the development process at any time proceeds in a direction perpendicular to the current surface.

Real resist response curves, often deviate strongly from a simple linear behaviour. Exemplary, Figure 9 displays a measured resist response curve (where we have used the positive resist AZ 1518, where the bleaching effect at moderate doses is negligible, combined with developer AZ 303, and a recording wavelength $\lambda_{\text {rec }}=442 \mathrm{~nm}$ ). The curve possesses a distinct threshold at $F_{0}=35 \mathrm{~mJ} / \mathrm{cm}^{2}$ followed by a nonlinear characteristics.

On the basis of this resist response curve the time evolution of the profile structure is simulated for two different exposure energy densities using Eq. (10). In the first case, the energy density is selected to be slightly above the threshold $\left(F_{\text {mod } 1}=F_{0}+20 \mathrm{~mJ} / \mathrm{cm}^{2}\right)$. The second value is near the saturation transition $\left(F_{\bmod 1}=F_{0}+200 \mathrm{~mJ} / \mathrm{cm}^{2}\right)$. Figure 10 shows the simulated results.

The calculated structures deviate significantly from the ideal sine profile corresponding to a constant duty cycle of $f=0.5$. In essence, the perfect sinusoidal form is only maintained for very flat profiles $(h<0.1 \mathrm{~g})$ together with a linear resist response, i.e. for weak exposure energies and only for one sin- 


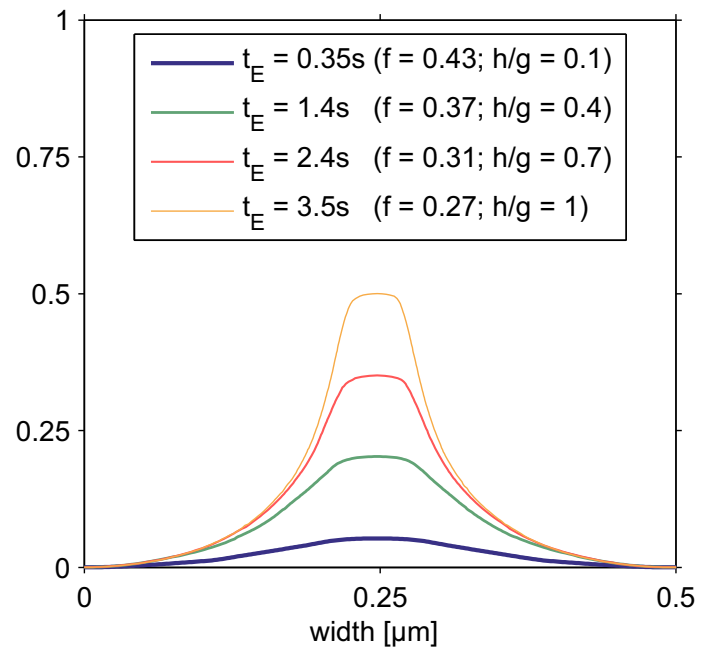

FIG. 10b Profile shapes obtained for four development times and two different energy doses: $200 \mathrm{~mJ} / \mathrm{cm}^{2}$ with increasing development time $t_{E}$ (from lower to upper curves).

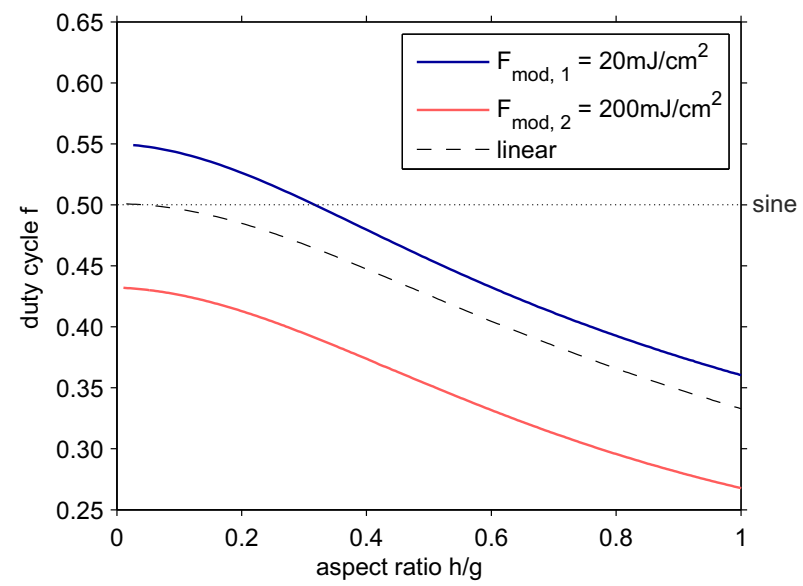

FIC. 11 Reduction of duty cycle $f$ with increasing aspect ratio of the profile for the nonlinear response in Figure 9 and the two different energy doses in Figure 10 obtained by the resist simulation. Dashed line: linear response curve for comparison.

gle height. In the presence of a nonlinear resist response, deviations from the perfect sinusoidal shape occur inevitably. For comparable structure heights the crests become more slender with increasing energy density. Figure 11 shows the calculated duty cycles in dependence of the aspect ratio for both exposure energies.

For each exposure energy, the duty cycle dependency $f=f(h / g)$ reveals a similar behaviour. The main difference is that the curves are vertically shifted. As an immediate consequence, the mere stretching of a unified profile is inappropriate with respect to an efficiency optimization.

On the basis of this resist simulation model, we start our theoretical calculations and use the real profile structures as an input in our theoretical efficiency optimizations. Both the profile height $h$ and the duty cycle $f$ are key parameters. From the resist simulation model we first generate a parameterdependent 2-dimensional profile matrix $y(x)[h, g]$, which we then feed into a downhill-simplex-algorithm [10] for the in- tegral equation method as the rigorous solver [9]. To ensure that the parameter dependence is captured with sufficient accuracy, our profile matrix consists of $100 \times 100$ elements each containing a whole profile curve $y(x)$. Instead of a direct optimum search within these $100 \times 100$ profiles, we exploit a discrete version with integer matrix indexing together with a bilinear interpolation, so that the algorithm converges very rapidly, despite the large set of data.

Regarding polychromatic spectrometer applications, we optimize the profile structure to achieve an efficiency optimum integrated over the entire wavelength range of interest, i.e. visible spectrum $400-700 \mathrm{~nm}$. In the Littrow mount for reflection geometry, the optimum height of an ideal sine grating turns out to be $h=190 \mathrm{~nm}(h / g=0.38)$ with an average diffraction efficiency over the wavelength range of about $\eta=65 \%$ (Figure 12(a)). However, the holographic profile yields a higher efficiency for the same profile height at each wavelength (Figure 12(b)). If we now determine the actual optimum height for the holographic profile we find $h=275 \mathrm{~nm}$, which corresponds to an aspect ratio of $h / g=0.55$ with $f=0.35$. Here, the integrated efficiency has even further increased, though there is a drop at the shorter wavelength end. If one compares this result with the equivalent ideal sine profile of height $275 \mathrm{~nm}$, one finds that the efficiency is extremely low (Figure 12(b)).

While for the holographic sine the efficiency increases with a higher and slender profile shape, it decreases for the corresponding ideal sine due to the fixed duty cycle. So optimization of ideal versus real holographic sine structures may go in completely opposite directions. Hence, for a correct optimization, the consideration of the actual development process is crucial. An approximation of real sine profile structures by their ideal counterparts will result in an increasing deviation of the efficiency performance with increasing aspect ratio. Moreover, because of the additional degree of freedom provided by the duty cycle, a slender holographic sinusoidal-like structure achieves an even higher efficiency.

The predicted profile structures for sinusoidal-like gratings are now compared to real grating structures that we have manufactured using the IL process as described above. For two different development times of $10 \mathrm{~s}$ and $20 \mathrm{~s}$ we have realized profiles with various heights and obtained the correlation of the duty cycle and the aspect ratio resulting from our experiments as displayed in Figure 13. The profile shapes have been measured using an atomic force microscope (AFM).

The dependence of the duty cycle on the aspect ratio of holographic sinusoidal-like gratings nearly follows the predicted values as far as the negative slope is concerned (Figure 13(a)). However, there is a larger offset for the duty cycles. So the resist simulation model seems to fail to predict the correct dependency. The reason for this turns out to be an inaccurate resist response curve. We presume that there was an aging of the resist, which leads to a reduced photosensitivity of the polymer. To account for this effect, the theoretical energy dose in the resist simulation model should be reduced, which then yields the solid lines in Figure 13(b). The slope is given through the orthogonal development with respect to the resist surface rather than through the shape of the resist curve 


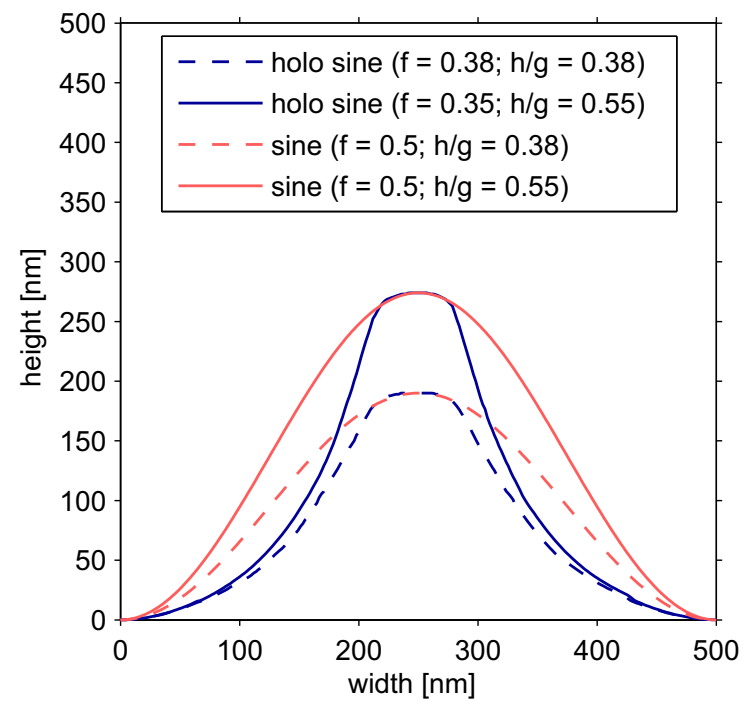

FIG. 12a (a) Specific profile shapes and (b) their corresponding diffraction efficiencies in Littrow mount comparing sine gratings with holographic sinusoidal gratings.

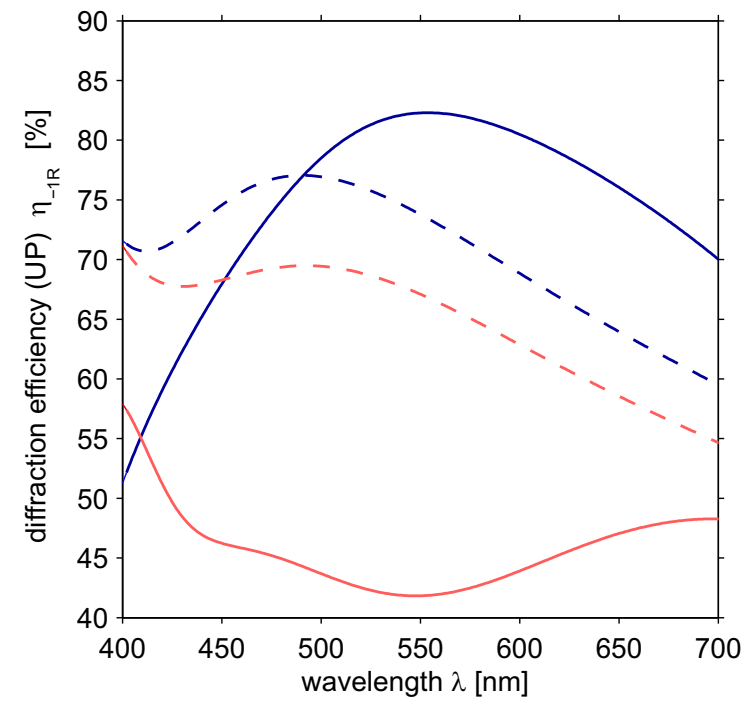

FIC. 12b (a) Specific profile shapes and (b) their corresponding diffraction efficiencies in Littrow mount comparing sine gratings with holographic sinusoidal gratings.

itself as can be seen from the linear fits in Figure 13(a). The resist curve only has a significant influence on the shift of the abscissa. The resist model requires an accurate knowledge of the resist response and it would require an exact measurement of the resist curve before each IL process, which is practically unfeasible.

Under the assumption of a linear resist response one finds that for any energy dose the solubility distribution is sinusoidal and that the profile shape - independently of the development time - results in the same dependence on the duty cycle on the aspect ratio as it is the case for the nonlinear resist response. Figure 10 reveals a clear dependence on the development time, which leads to slender profiles for higher doses

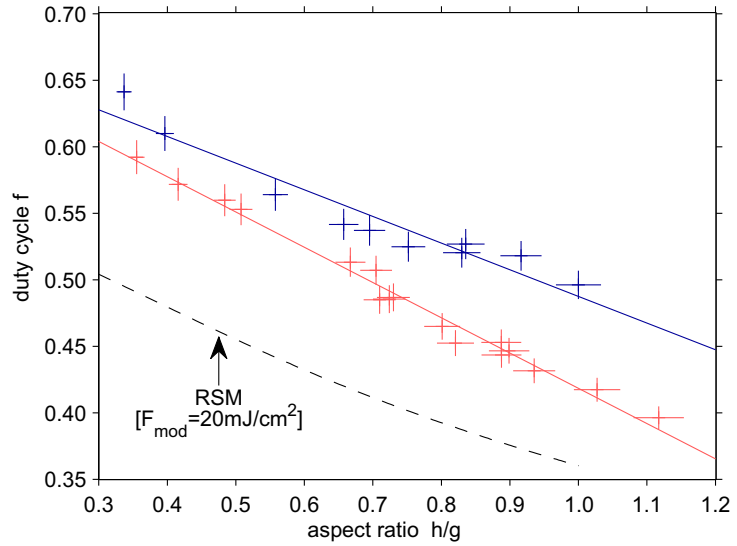

FIG. 13a Experimentally obtained grating parameters (crosses). Comparison with (a) the RSM-model as discussed above.

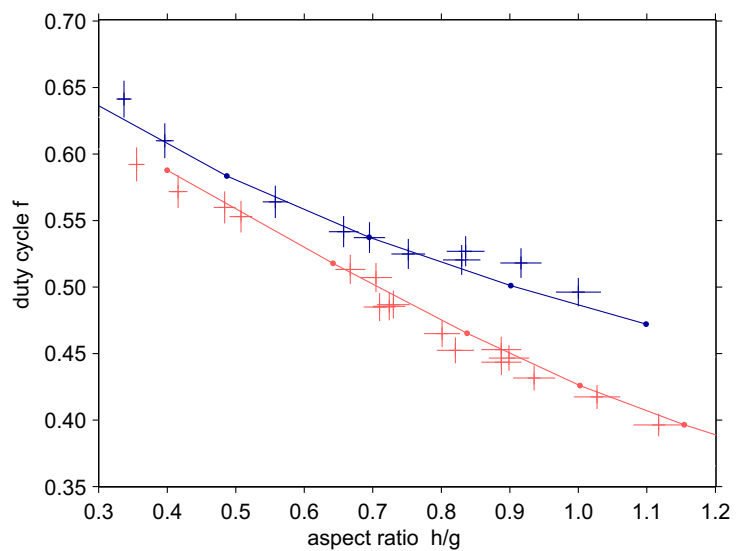

FIG. 13b Experimentally obtained grating parameters (crosses). Comparison with the RSM model modified to account for actual energy doses due to the aging of the resist.

if the same height is achieved, and consequently the underlying resist curve must have been nonlinear.

\subsection{Characterization and inverse reconstruction of holographic structures}

For many applications it would be very beneficial to get the topographical information about the real diffractive structure from the measurement of the optical efficiency performance. Rigorous grating analysis determines how a certain profile structure translates into the optical performance. We now want to turn the question the other way round: what structure corresponds to a certain optical efficiency performance achieved? This leads in general to the inverse grating problem. We exploit a procedure from scatterometry and use the optical performance to determine the values of the free parameters of the profile shape.

This procedure is supported and facilitated by the concentration in the description of the real possible topography to a specific analytical model. From AFM measurements, it has become clear (cf. Figure 10), that the manufactured holographic 


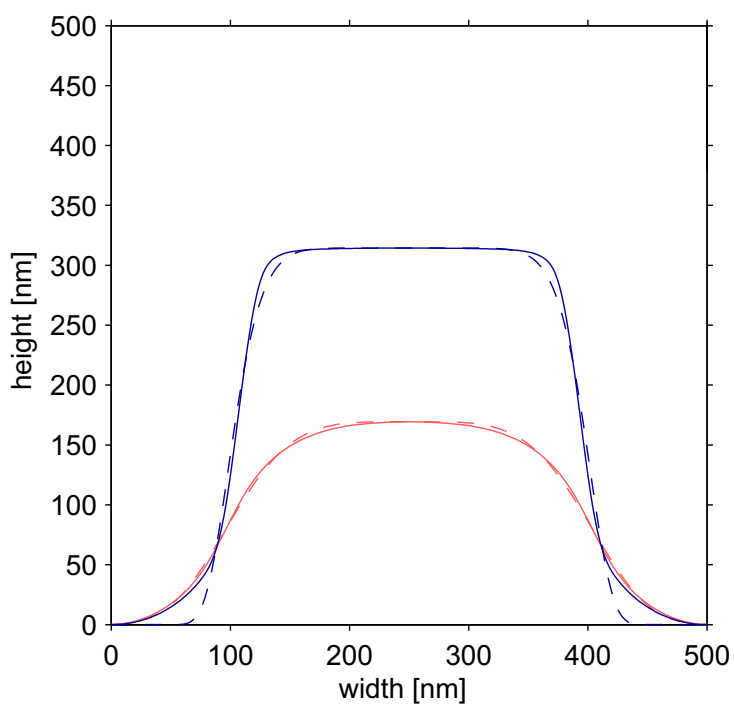

FIG. 14a Inverse reconstruction of two typical holographic grating structures. (a) Profile shapes and (b) diffraction efficiencies. Solid lines: direct efficiency calculation from AFM-profiles. Dashed line: reconstructed profiles from efficiencies using superGaussian profiles.

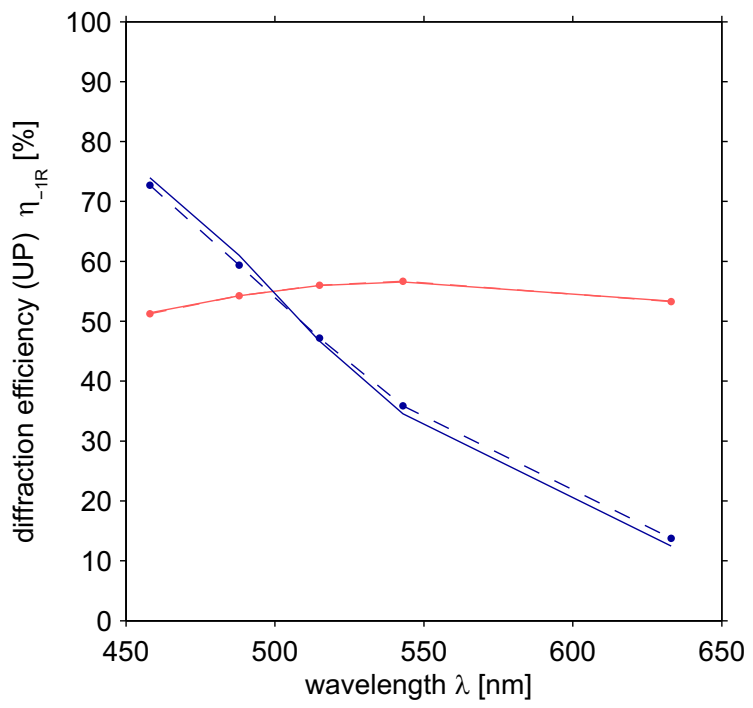

FIC. $14 \mathrm{~b}$ Inverse reconstruction of two typical holographic grating structures. (a) Profile shapes and (b) diffraction efficiencies. Solid lines: direct efficiency calculation from AFM-profiles. Dashed line: reconstructed profiles from efficiencies using superGaussian profiles.

profile shapes can be approximated by super-Gaussians defined as

$$
y(x)=h \times \exp \left(-\left|\frac{2 x-g}{\sigma \times g}\right|^{n} \times \ln 2\right),
$$

where the height $h$ and grating period $g$ are scaling factors in the $x$ - and $y$-direction, resp., similar to the case of ideal structures. The super-Gaussian order $n$ and the profile width $\sigma$, in addition, are key parameters, which allow us to account for the specific features of the holographic profiles, such as rounded edges and the variation in the duty cycle $f$, e.g. AFM measurements of manufactured structures show a good corre- lation to the topographic assumptions (Figure 14(a)). A variation of the illumination parameters shows the appearance of two types of profile geometries: one shallow profile with soft edges (red curves), and the other with a large height and pronounced steep edges together with a dominant flat top (solid blue lines in Figure 14(a)). A forward calculation of the efficiency from the topographic data for both extreme geometries is depicted in Figure 14(b) (solid lines). In the backward approach, the calculated profile shape from the efficiency data shows a highly correspondent calculated surface profile to the measured topography. We have fitted the profile using a least square minimization algorithm:

$$
\begin{aligned}
& \sum_{M S E}(h, f, n) \\
& =\frac{1}{5} \sum_{i=1}^{5}\left[\left(\eta_{T E}^{\text {meas. }}\left(\lambda_{i}\right)-\eta_{T E}^{I E S M P}\left(\lambda_{i}, h, f, n\right)\right)^{2}\right. \\
& \left.\quad+\ldots+\left(\eta_{T M}^{\text {meas. }}\left(\lambda_{i}\right)-\eta_{T M}^{\text {IESMP }}\left(\lambda_{i}, h, f, n\right)\right)^{2}\right] .
\end{aligned}
$$

The inverse reconstruction (dashed lines) starts from the optical performance at 5 selected laser wavelengths: $457.9 \mathrm{~nm}$, $488.0 \mathrm{~nm}, 514.5 \mathrm{~nm}, 543.5 \mathrm{~nm}$ and $632.8 \mathrm{~nm}$ (dots in Figure 14(b)). Varying $\sigma(f, h)$ and $n$ the algorithm searches for the super-Gaussian profile with the least deviation from the rigorous efficiency for all 5 wavelengths.

The profile shapes that we find (dashed lines) are in good agreement with the measurements (solid). Though, for the steep profile type, there are structural discrepancies found at the upper edges and the lower sockets (Figure 14(a)). Yet, the optical performance displays only very weak deviations (blue curves in Figure 14(b)). That means the assumption of a superGaussian profile shape of a real manufactured holographic structure allows to predict the diffraction efficiency and vice versa, with the measured diffraction efficiency it is possible to get information about the profile shape.

\section{CONCLUSIONS}

We have employed a lithography model and have successfully been able to predict the efficiency performance of real holographic sine and sinusoidal-like structures. Varying the profile height and the period will change the specific profile shape due to the manufacturing process, which we have taken into account during the optimization exploiting a twodimensional profile matrix. According to a two-wave theory, in the resonance domain the profile shape has little influence on the maximum diffraction efficiency. Light sees only the first Fourier component of the profile. Rounded edges, as for example occuring in interference lithography, have only little influence on the maximum efficiency here. However, we have found that the spectrally broadband efficiency is higher for sinusoidal-like profiles that can be accurately modelled by super-Gaussian shapes. So in the resonance domain optimized holographic sine gratings may achieve a higher efficiency performance than the corresponding optimized ideal sine and can compete with sawtooth profiles. Analogously, we are convinced that holographic blaze profiles show a similar relation to their ideal counterpart, but the corresponding lithography model is much more advanced and more sensi- 
tive to the experimental parameters. It has to be left for future work.

\section{References}

[1] R. Brunner, M. Burkhardt, N. Correns, and K. Rudolf, "Microspectrometer based on holographically recorded diffractive elements using supplementary holograms" Opt. Express 16, 12239-12250 (2008).

[2] M. D. Missig, and G. M. Morris, "Diffractive optics applied to eyepiece design" Appl. Optics 34, 2452-2461 (1995).

[3] W. Knapp, G. Blough, K. Khajurival, R. Michaels, B. Tatian, and B. Volk, "Optical design comparison of $60^{\circ}$ eyepieces: one with a diffractive surface and one with aspherics" Appl. Optics 36, 4756-4760 (1997).

[4] R. Brunner, R. Steiner, K. Rudolf, and H.-J. Dobschal, "Diffractiverefractive hybrid microscope objective for $193 \mathrm{~nm}$ inspection systems" Proc. SPIE 5177, 9-15 (2003).

[5] R. Brunner, R. Steiner, H.-J. Dobschal, D. Martin, M. Burkhardt, and M. Helgert, "New solutions to realize complex optical systems by a combination of diffractive and refractive optical components" Proc. SPIE 5183, 47-55 (2003).

[6] R. Brunner, M. Burkhardt, A. Pesch, 0. Sandfuchs, M. Ferstl, S. C. Hohng, and J. 0. White, "Diffraction based solid immersion lens" J. Opt. Soc. Am. A 21, 1186-1191 (2004).

[7] 0. Sandfuchs, R. Brunner, D. Pätz, S. Sinzinger, and J. Ruoff, “Rigorous analysis of shadowing effects in blazed transmission gratings" Opt. Lett. 31, 3638-3640 (2006).

[8] 0. Sandfuchs, A. Pesch, and R. Brunner, "Rigorous modeling of dielectric and metallic blaze gratings in the intermediate structure regime" Proc. SPIE 6675, 667501-1-667501-8 (2007).
[9] A. Rathsfeld, G. Schmidt, and B. H. Kleemann, "On a fast integral equation method for diffraction gratings" Commun. Comput. Phys. 1, 984-1009 (2006).

[10] J. Nelder, and R. Mead, "A simplex method for function minimization." Comput. J. 7, 308-313 (1965).

[11] M. Burkhardt, and R. Brunner, "Functional integrated optical elements for beam shaping with coherence scrambling property, realized by interference lithography" Appl. Optics 46, 7061-7067 (2007).

[12] E. G. Loewen, and E. Popov, Diffraction gratings and applications (Marcel Dekker, Inc., New York, 1997).

[13] B. de A. Mello, I. F. Da Costa, C. R. A. Lima, and L. Cescato, "Developed profile of holographically exposed photoresist gratings" Appl. Optics 34, 597-603 (1995).

[14] M. A. Golub, and A. A. Friesem, "Analytic design and solutions for resonance domain diffractive optical elements" J. Opt. Soc. Am. A 24, 687-695 (2007).

[15] 0. Sandfuchs, D. Pätz, S. Sinzinger, A. Pesch, and R. Brunner, "Analysis of the influence of the passive facet of blazed transmission gratings in the intermediate diffraction regime" J. Opt. Soc. Am. A 25, 1885-1893 (2008).

[16] H. Kogelnik, "Coupled wave theory for thick hologram gratings" Bell Syst. Tech. J. 48, 2909-2947 (1969).

[17] H. J. Gerritsen, D. K. Thornton, and S. R. Bolton, "Application of Kogelnik's two-wave theory to deep, slanted, highly efficient, relief transmission gratings" Appl. Optics 30, 807-814 (1991). 